\title{
Mixed QCD-EW Corrections to Yukawa Coulings in the SM and the MSSM
}

\section{Luminita Mihaila*}

Institut for Theoretical Physics, University of Heidelberg, 69117 Heidelberg, Germany

E-mail: mihailaethphys.uni-heidelberg.de

\begin{abstract}
We report in this presentation on the recent calculations of the mixed QCD-EW corrections to the top- and bottom-Yukawa couplings within the Standard Model (SM) and its minimal supersymmetric extension (MSSM). We concentrate on the comparison between the SM and the MSSM predictions and on the non-decoupling behavior of the SUSY corrections that might open a new window to indirect searches for new physics.
\end{abstract}

13th International Symposium on Radiative Corrections 24-29 September, 2017

St. Gilden, Austria

\footnotetext{
* Speaker.
} 


\section{Introduction}

With the Higgs boson discovery, particle physics entered a new era of tremendous intensity of detailed and careful study of its properties. Hopefully, accurate understanding of the Higgs phenomenology together with new information from experiments at the LHC will provide us a tool for exploring new physics.

Great interest is currently devoted to the study of the Higgs boson couplings to the electroweak gauge bosons $W$ and $Z$ and to the top- and bottom-quarks or tau-leptons. Deviations in these couplings could possibly be observed once the currently large uncertainties will be improved as part of the LHC program and at a future Higgs factory. It has been shown that the Higgs couplings will be sensitive to new physics at the multi-TeV scale once percent level precision on coupling measurements will be achieved (for a recent review see [1]).

One of the most studied models for describing physics beyond the SM is its minimal supersymmetric extension (MSSM). However, after the end of the LHC run I and the start of the LHC run II, the direct searches for supersymmetric particles remained unsuccessful, thus increasing the exclusion bounds for the supersymmetric mass scale towards the TeV range. For the time being, it seems that if supersymmetry is realised in high energy physics the most likely scenario is the one where all SUSY particles are much heavier than the SM ones. For such a mass hierarchy (also called the "decoupling limit"), it was shown [2] that the effects of the heavy particles on physical observables measured in the low-energy regime scales like $M_{\mathrm{EW}}^{2} / M_{\mathrm{SUSY}}^{2}$. This conclusion is just a consequence of the Appelquist-Carrazone decoupling theorem [4], that states that the effects of the heavy fields are power suppressed at low momenta. The effect of loops of heavy particles is that the parameters, e.g. masses, couplings and mixing angles, of the low-energy theory have different values from those in the underlying theory. Since the renormalized couplings have no particular value, the heavy particles are unobservable until close to the threshold. Therefore, for very heavy SUSY scales, deviations from the SM predictions of observables will be very challenging to detect. In such a case, additional efforts both in theory and experiment are required to identify physical observables for which the power suppressed behaviour does not occur (i.e. the Appelquist-Carrazone theorem is violated), or the decoupling limit is delayed by parametric enhancements like in the presence of large $\tan \beta$ values [5]. Prominent examples for this class of observables are the mass of the lightest Higgs boson in SUSY and its self coupling in BSM theories [6, 7] that receives radiative corrections increasing with the heavy mass scales and their mixing parameters. Another possibility to search for BSM effects is to relate parameters measured at low scales, for which violation of decoupling theorem occurs, to those of the full theory via renormalization group (RG) methods and study their effects on physical observables.

Let us stress that the use of RG methods together with the decoupling effects is the only possibility to derive the input parameters for the full theory without breaking the convergence of the perturbation theory due to the occurrence of large logarithms. We give below an explicit example concerning the determination of the running top quark mass in the MSSM. It is also usually necessary to work at higher oder in perturbation theory to keep the theoretical uncertainties below the experimental precision.

In the present study $[12,13]$ we study the the running of the top quark mass and Yukawa couplings with the energy in the decoupling limit of the MSSM. Under the decoupling limit we 
understand that all the superpartners, including the additional Higgs particles $A, H^{0}, H^{ \pm}$, are much heavier than the SM particles. We assume here that the relation $M_{\mathrm{SUSY}}, M_{\mathrm{A}} \gg M_{\mathrm{EW}}$ holds. The case for an intermediate Higgs sector ( $M_{\mathrm{SUSY}} \gg M_{\mathrm{A}} \gg M_{\mathrm{EW}}$ ), i.e at intermediate energies the $2 \mathrm{HDM}$ is at work, was studied in detail in the literature $[5,8,9,10,11]$. Here, we consider the case for which the lightest Higgs boson behaves SM like and no additional particles have masses at intermediate energy scales.

The non-decoupling effects for the running quark masses and Yukawa couplings increase logarithmically with the SUSY mass scale and have a polynomial dependence with maximal degree three on the mixing parameters in the squark sectors. From a phenomenological point of view, the non-decoupling effects are usually few times larger than the current accuracy on the bottom and top quark mass determinations. For Yukawa couplings the effects are in the range of precision expected for a $100 \mathrm{TeV}$ collider. In order to keep the theoretical uncertainties well below the experimental accuracy, we consider here the two-loop radiative corrections of order $\mathscr{O}\left(\alpha_{s}^{2}, \alpha_{s} \alpha_{t}\right)$ induced by the strong and the top-Yukawa couplings, and neglect the bottom-Yukawa coupling except for scenarios with large $\tan \beta$ values.

\section{Framework}

For the study of the running quark masses and Yukawa couplings up to $\mathscr{O}\left(\alpha_{s}^{2}, \alpha_{s} \alpha_{t}\right)$ it is sufficient to study the following three operators

$$
\mathscr{L}_{\text {eff }}=\mathscr{L}_{\mathrm{SM}}^{(6)}+\mathscr{L}_{\mathrm{eff}}^{h}+\ldots ; \quad \mathscr{L}_{\mathrm{eff}}^{h}=-\frac{h^{(0)}}{v^{(0)}}\left[C_{1}^{0} \mathscr{O}_{1}^{0}+\sum_{q=u, d, \ldots}\left(C_{2 q}^{0} \mathscr{O}_{2 q}^{0}+C_{3 q}^{0} \mathscr{O}_{3 q}^{0}\right)+\ldots\right]
$$

Here $\mathscr{L}_{\mathrm{SM}}^{(6)}$ denotes the usual SM Lagrangian with six active quark flavours but without the Yukawa sector. The latter is considered explicitely in $\mathscr{L}_{\text {eff }}^{h}$, that collects all interactions with just one Higgs field. The ellipsis stand for the remaining higher-dimensional operators that we do not study here. The Wilson coefficients $C_{i}, i=1,2 q, 3 q$, parameterize the effects of the heavy particles and the superscript 0 labels bare quantities. The three operators relevant in our computation are defined as

$$
\begin{aligned}
& \mathscr{O}_{1}^{0}=\left(G_{\mu, v}^{0, \prime, a}\right)^{2} \\
& \mathscr{O}_{2 q}^{0}=m_{q}^{0, \prime} \bar{q}^{0, \prime} q^{0, \prime} \\
& \mathscr{O}_{3 q}^{0}=\bar{q}^{0, \prime}\left(i p^{0, \prime}-m_{q}^{0, \prime}\right) q^{0, \prime}
\end{aligned}
$$

where $G_{\mu, v}^{0, \prime, a}$ and $D_{\mu}^{0, \prime}$ are the gluon field strength tensor and the covariant derivative for quark fields, and the primes label the quantities in the effective theory. The operator $\mathscr{O}_{3 q}$ vanishes by the fermionic equation of motion and it will not contribute to physical observables. So, the last term in Eq. (2.1) might be omitted, once the coefficients $C_{1}^{0}, C_{2 q}^{0}$ are determined. In the decoupling limit $C_{1} \rightarrow 0$ and $C_{2 q} \rightarrow 1$ and the higher-dimensional operators decouple. We verified through an explicit calculation that the higher-dimensional operators are power suppressed at one and two loops in the top sector.

In physical renormalization schemes the decoupling of the heavy particles is manifest. Explicitly, the low-energy theory is simply constructed by ommiting the heavy fields without changing 
the parameters of the theory related with the light degrees of freedom. In mass independent renormalization schemes the Appelquist-Carrazone decoupling theorem does not hold and the Green functions usually depend on the heavy masses and mixing parameters present in the full theory. In order to avoid potentially large logarithms and power corrections one has to decouple by hand the heavy particles from the theory. Since different renormalization prescriptions differ only by a reparametrization, one can render the Green functions to be equal in the two schemes by a redefinition of the parameters and fields present in the effective theory. The reparametrization relations between the parameters defined in the full and effective theories are denotes as decoupling or matching relations and they can be computed order by order in perturbation theory. We calculated the decoupling coefficients for the quark masses and the third generation Yukawa-coupling at $\mathscr{O}\left(\alpha_{s}^{2}, \alpha_{s} \alpha_{t}\right)$.

The explicit computation of the decoupling coefficients can be reduced to the calculation of Green functions containing at least one heavy line within the loops in the limit of vanishing external momenta and light particle masses. These very strong simplifications can be applied because the infrared singularities cancel when the high-energy and the low-energy are matched [2]. From the technical point of view, the calculation of decoupling coefficients is reduced to massive tadpole topologies. The later are known analytically at two loop order and can be derived with the help of asymptotic expansions up to four loops.

\section{Results}

In this section, we review the phenomenological implications of the two-loop the decoupling coefficients. Two-loop decoupling effects are usually combined with three-loop renormalization group equations (RGEs) in order to properly resum the logarithms that arise in the calculation.

In Fig. 1 we present the running top-quark mass in the full theory (in the "heavy sfermion" scenario) as a function of the renormalization scale $\mu_{\text {ren }}$ at which it is evaluated. The black (middle) curves display the results obtained with the running and decoupling method (that we refer at as the decoupling method and is denoted as "DEC" in the legend of the plot) at one (dotted line) two (dashed line) and three (full line) loops. The blue curves show the predictions obtained directly via the ratio between the top-quark pole and the running masses in the SUSY-QCD, using the code TS IL [15]. The dashed line corresponds to the one-loop results and the full line displays the twoloop contributions. As can be seen from the figure, the radiative corrections for the decoupling method are very small (tenth of $\mathrm{MeV}$ between one- and two-loop order contributions and negligible at the three loops) as compared with the current experimental uncertainty on the top quark pole mass of about $1 \mathrm{GeV}$. The perturbative series is very well converging and the contributions from the unknown higher order corrections are negligible for all renormalization scales. The radiative corrections obtained via the direct method are much larger than the experimental uncertainty. Even at the two-loop order, they amount to $10 \mathrm{GeV}$ at the electro-weak scale and increase further at renormalization scales comparable with the squark masses. In this case higher order contributions are necessary to bring the theoretical precision at the same level as the experimental one. One observes also that the predictions obtained in the two methods at the two-loop order agree very well for small renormalization scales below $400 \mathrm{GeV}$. This can be explained by the fact that in this case the logarithmic contributions (of the form $\ln \left(M_{\text {top }} / \mu\right)$ ) are small and the resummation gives only 


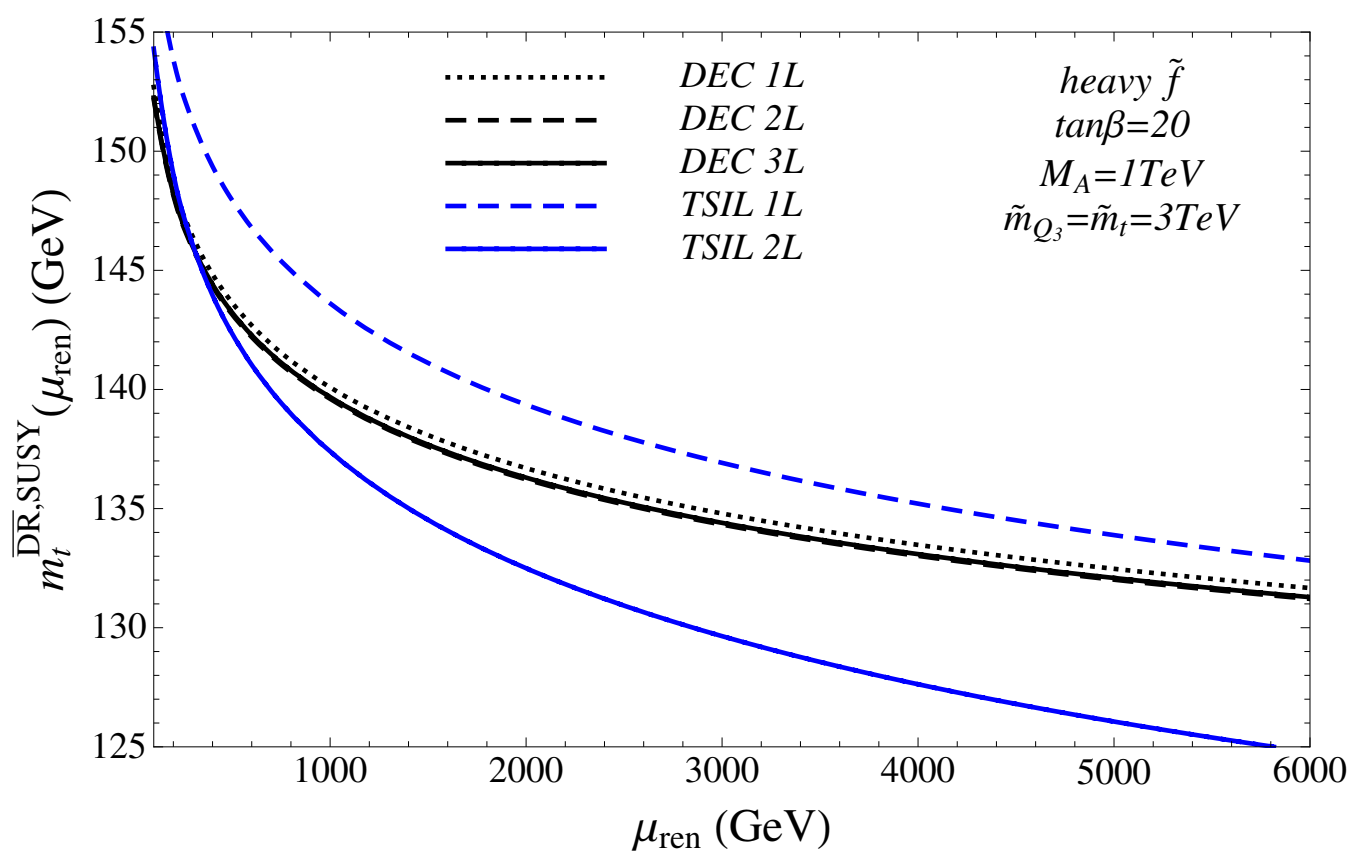

Figure 1: Renormalization scale dependence of the running top quark mass for the "heavy sfermion" scenario. The black curves show the results obtained within the decoupling method at one (dotted line), two (dashed line) and three (full line) loops. The blue lines display the results obtained with the code TSIL at one (dashed line) and two (full line) loops.

minor corrections. When the running top-quark mass is evaluated at high energy scales the resummation of the large logarithms becomes important and the difference between the two predictions can reach about $10 \mathrm{GeV}$. Let us also point out that in the domain where the resummation is expected to bring only small effects the differences between the two methods decrease considerably when going from one to two loops as is expected in perturbation theory.

We show in Fig. 2 the scale evolution of $m_{t}$ and $\alpha_{t}$ both in the SM and the MSSM for the pMSSM scenario, where the matching between the two theories was performed at $\mu_{\mathrm{dec}}=400 \mathrm{GeV}$. Namely, below $400 \mathrm{GeV}$ the SM is considered as the theory describing the physical phenomena. Above this energy scale, the MSSM is the underlying theory. For the dot-dashed lines, only the QCD (below $\mu_{\mathrm{dec}}=400 \mathrm{GeV}$ ) and SUSY-QCD (above $\mu_{\mathrm{dec}}=400 \mathrm{GeV}$ ) contributions are taken into account. For the one-loop running (dotted lines in the plots) only a change of slopes occurs, because no decoupling is taken into account. At two and three loops, the running is accompanied by one- and two-loop decoupling. The vertical shifts at $\mu_{\mathrm{ren}}=\mu_{\mathrm{dec}}=400 \mathrm{GeV}$ display directly the effects of the decoupling procedure. The numerical values of the mixed (SUSY)QCD-Yukawa contributions at three loops can be read from the difference between the full and the dot-dashed curves in the figure. As already pointed out, their magnitude depends on the scale choice and are phenomenologically significant for the top sector of the MSSM. The magnitude of the $\mathscr{O}\left(\alpha_{s} \alpha_{t}\right)$ corrections to the decoupling coefficients for $m_{t}$ and $\alpha_{t}$ can be read from the difference between the solid and the large dotted lines. For the MSSM scenario under consideration, they are much smaller than the genuine QCD corrections, that are however comparable with the experimental 


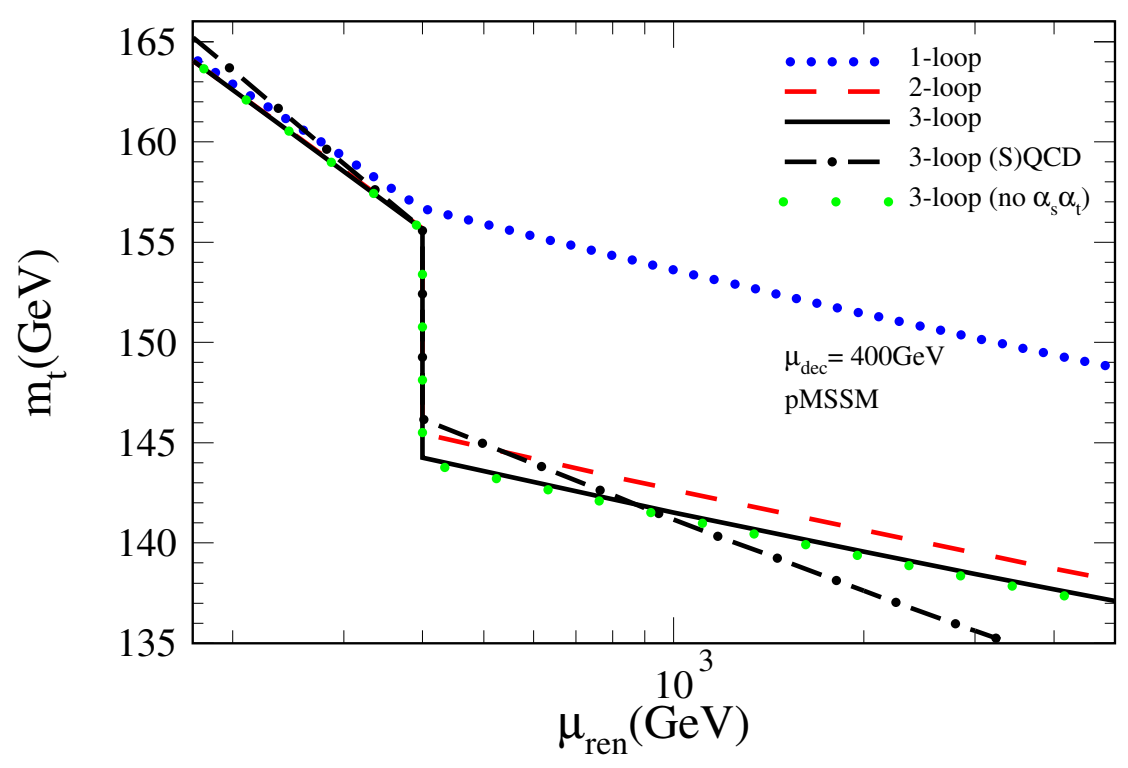

(a)

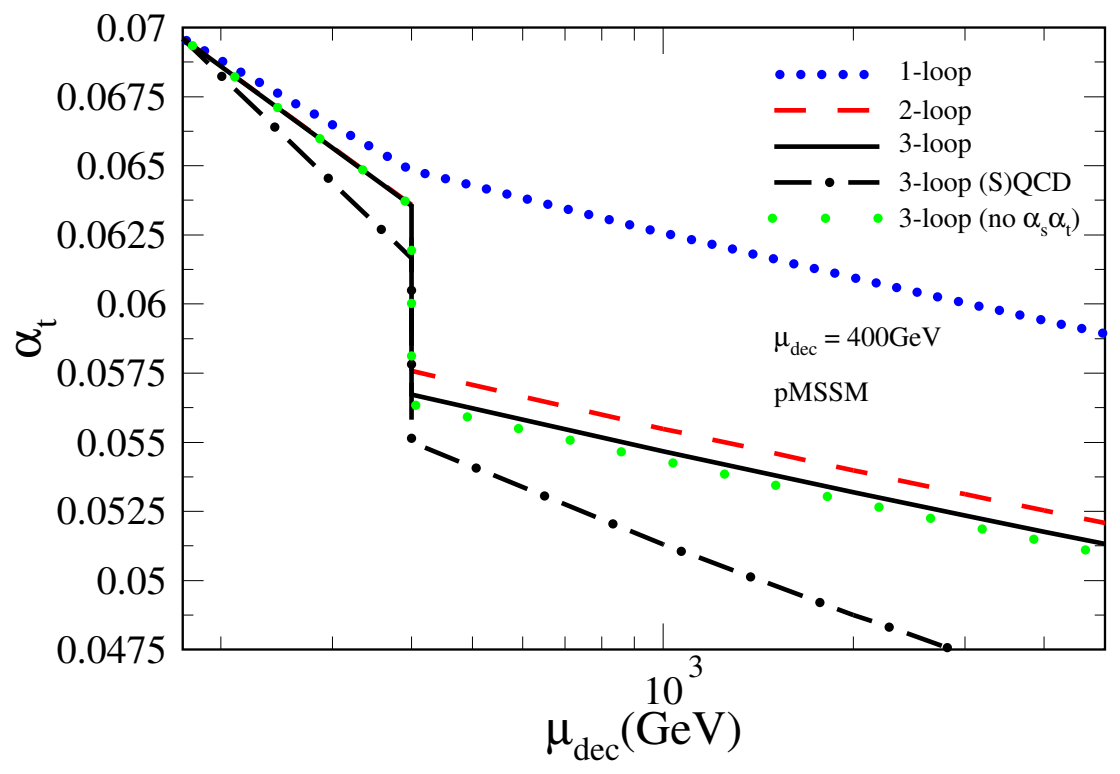

(b)

Figure 2: Running of $m_{t}$ and $\alpha_{t}$. The matching scale between the SM and the MSSM is $\mu_{\mathrm{dec}}=$ $400 \mathrm{GeV}$. The dotted, dashed and full lines correspond to one-, two- and three-loop running. The dot-dashed lines display the three-loop SUSY-QCD contributions. The large dotted lines marked in the legend with the label (no $\alpha_{s} \alpha_{t}$ ) show the predictions of the three-loop analysis, where the $\mathscr{O}\left(\alpha_{s} \alpha_{t}\right)$ contributions to the decoupling coefficients for $m_{t}$ and $\alpha_{t}$ were excluded. The jumps at $\mu_{\mathrm{dec}}=400$ display the decoupling effects. 
precision on the top pole mass $M_{\mathrm{top}}$.

\section{Conclusions}

Although the running masses and couplings are themselves not physical observables, they are necessary ingredients for the theoretical predictions of cross sections, branching ratios or physical masses. For example, if in a diagrammatic calculation performed within the MSSM at fixed order or in the EFT approach the running top-quark mass and/or running top-Yukawa and strong couplings are used, then the non-decoupling effects we discussed here have to be considered when the numerical values of the running input parameters are calculated. From our numerical analysis it turned out that the genuine $\mathscr{O}\left(\alpha_{s} \alpha_{t}\right)$ contributions to the matching coefficients are well below the experimental precision. However, the complete $\mathscr{O}\left(\alpha_{t}, \alpha_{s} \alpha_{t}\right)$ corrections (including the RGE and the threshold effects) to the running top quark mass amount to about few $\mathrm{GeV}$ and for the topYukawa coupling reach the percent range. The $\mathscr{O}\left(\alpha_{s} \alpha_{t}\right)$ corrections to the decoupling coefficients for $m_{t}$ and $\alpha_{t}$ are necessary, for example, for the prediction of the lightest Higgs boson mass in supersymmetric theories and for the vacuum stability studies in such theories, when going beyond two-loop accuracy.

\section{References}

[1] C. Englert, A. Freitas, M. M. Mühlleitner, T. Plehn, M. Rauch, M. Spira and K. Walz, Precision Measurements of Higgs Couplings: Implications for New Physics Scales, J. Phys. G 41 (2014) 113001 [arXiv:1403.7191 [hep-ph]].

[2] J. C. Collins, Renormalization : An Introduction to Renormalization, The Renormalization Group, and the Operator Product Expansion, Cambridge University Press, 1986.

[3] A. Dobado, M. J. Herrero and S. Penaranda, The Higgs sector of the MSSM in the decoupling limit, Eur. Phys. J. C 17 (2000) 487 [hep-ph / 0002134 ]; H. E. Haber, M. J. Herrero, H. E. Logan, S. Penaranda, S. Rigolin and D. Temes, Decoupling properties of MSSM particles in Higgs and top decays, [hep-ph/0102169].

[4] T. Appelquist and J. Carazzone, nfrared Singularities and Massive Fields, Phys. Rev. D 11 (1975) 2856.

[5] L. J. Hall, R. Rattazzi and U. Sarid, The Top quark mass in supersymmetric SO(10) unification Phys. Rev. D 50 (1994) 7048; M. S. Carena, D. Garcia, U. Nierste and C. E. M. Wagner, Effective Lagrangian for the $\overline{\mathrm{t}} b \mathrm{H}^{+}$interaction in the MSSM and charged Higgs phenomenology Nucl. Phys. $B$ 577 (2000) 88 [arXiv: hep-ph/9912516].

[6] P. H. Chankowski, S. Pokorski and J. Wagner, (Non)decoupling of the Higgs triplet effects, Eur. Phys. J. C 50 (2007) 919 [hep-ph/0605302].

[7] S. Kanemura, M. Kikuchi and K. Yagyu, Fingerprinting the extended Higgs sector using one-loop corrected Higgs boson couplings and future precision measurements, Nucl. Phys. B 896 (2015) 80 [arXiv:1502.07716 [hep-ph]].

[8] D. Noth and M. Spira, Supersymmetric Higgs Yukawa Couplings to Bottom Quarks at next-to-next-to-leading Order, JHEP 1106 (2011) 084 [arXiv: 1001.1935 [hep-ph] ]; D. Noth and M. Spira, Higgs Boson Couplings to Bottom Quarks: Two-Loop Supersymmetry-QCD Corrections, Phys. Rev. Lett. 101 (2008) 181801 [arXiv:0808.0087 [hep-ph]]. 
[9] L. Mihaila and C. Reisser, $O\left(\alpha_{s}^{2}\right)$ corrections to fermionic Higgs decays in the MSSM, JHEP 1008 (2010) 021 [arXiv:1007.0693 [hep-ph]].

[10] R. Harlander, L. Mihaila and M. Steinhauser, Two-loop matching coefficients for the strong coupling in the MSSM, Phys. Rev. D 72 (2005) 095009; [arXiv: hep-ph/ 0509048 ]; A. V. Bednyakov, Running mass of the b-quark in QCD and SUSY QCD, Int. J. Mod. Phys. A 22 (2007) 5245 [arXiv:0707.0650 [hep-ph]]; A. Bauer, L. Mihaila and J. Salomon, Matching coefficients for $\alpha_{s}$ and $m(b)$ to $O\left(\alpha_{s}^{2}\right)$ in the MSSM, JHEP 0902 (2009) 037 [arXiv: 0810.5101 [hep-ph]].

[11] A. V. Bednyakov, On the two-loop decoupling corrections to tau-lepton and b-quark running masses in the MSSM, Int. J. Mod. Phys. A 25 (2010) 2437 [arXiv:0912.4652 [hep-ph] ].

[12] D. Kunz, L. Mihaila and N. Zerf, $\mathscr{O}\left(\alpha_{S}^{2}\right)$ corrections to the running top-Yukawa coupling and the mass of the lightest Higgs boson in the MSSM, JHEP 1412 (2014) 136 [arXiv: 1409.2297 [hep-ph]].

[13] L. Mihaila and N. Zerf, $\mathscr{O}\left(\alpha_{s} \alpha_{t}\right)$ (non)decoupling effects within the top-sector of the MSSM, JHEP 1705 (2017) 019 [arXiv:1612.06619 [hep-ph] ].

[14] L. Mihaila, B. Schmidt and M. Steinhauser, $\Gamma(H \rightarrow b \bar{b})$ to order $\alpha \alpha_{s}$, Phys. Lett. $B 751$ (2015) 442 [arXiv:1509.02294 [hep-ph]].

[15] S. P. Martin and D. G. Robertson, TSIL: A Program for the calculation of two-loop self-energy integrals, Comput. Phys. Commun. 174 (2006) 133 [hep-ph/ 0501132 ]. S. P. Martin, Evaluation of two loop selfenergy basis integrals using differential equations, Phys. Rev. D 68 (2003) 075002 [hep-ph/0307101]. 